\title{
Phylum-targeted pyrosequencing reveals diverse planctomycete populations in a eutrophic lake
}

\author{
Blaire Steven ${ }^{1, *}$, Scot E. Dowd ${ }^{4}$, Kayley H. Schulmeyer ${ }^{1}$, Naomi L. Ward ${ }^{1,2,3}$ \\ ${ }^{1}$ Department of Molecular Biology, Dept 3944, 1000 E. University Ave, ${ }^{2}$ Department of Botany, and ${ }^{3}$ Program in Ecology, \\ University of Wyoming, Laramie, Wyoming 82071, USA \\ ${ }^{4}$ Research and Testing Laboratory, Lubbock, Texas 79407, USA
}

\begin{abstract}
Although populations of planctomycete bacteria are nearly ubiquitous in aquatic habitats, we lack a detailed understanding of their diversity and structure. This is due to difficulty in obtaining cultured representatives, but also to low recovery rates for planctomycete 16S rRNA genes when universal PCR primers are employed for sequencing studies. In an attempt to expand recoverable planctomycete diversity, we investigated the use of primers targeting the Planctomycetes phylum. Planctomycete populations present during an algal bloom in a eutrophic lake were characterized by clone library sequencing and pyrosequencing of 16S rRNA genes, using planctomycetetargeted primers. We analyzed samples recovered from the sediment, water column, and algal mats within the lake's littoral zone. Sequences related to 9 planctomycete genera were identified within the 6287 planctomycete sequences and 1730 operational taxonomic units (OTUs) recovered. We observed variation in the specificity of the planctomycete primers, with more non-planctomycete sequences recovered through pyrosequencing than through cloning-based sequencing. Nevertheless, the results of our study suggest that phylum-targeted pyrosequencing is a useful tool for better describing the diversity of bacterial sub-populations. This methodology could be employed for future testing of hypotheses regarding spatial and temporal differences in planctomycete diversity and abundance. Candidate hypotheses arising from this preliminary study include (1) members of certain planctomycete genera (particularly Rhodopirellula) are enriched in algal mats relative to other planctomycete genera; (2) sediments harbor the most diverse planctomycete populations; and (3) most planctomycete OTUs are not shared between lake habitats.
\end{abstract}

KEY WORDS: Planctomycetes $\cdot$ Freshwater lake $\cdot$ Algal bloom Resale or republication not permitted without written consent of the publisher

\section{INTRODUCTION}

The phylum Planctomycetes represents a widely distributed, but relatively understudied bacterial group. Planctomycete bacteria have been isolated from environments such as freshwater (Staley 1973, Franzmann \& Skerman 1984, Wang et al. 2002), ocean water (Schlesner \& Stackebrandt 1986), soil (Wang et al. 2002), and hot springs (Giovannoni et al. 1987, Kahan 1961). Culture-independent surveys have detected planctomycete bacteria in environments as diverse as human (Andersson et al. 2008) and termite (Köhler et al. 2008) guts, permafrost (Steven et al. 2007), and a wastewater treatment plant (Chouari et al. 2003). The detection of uncultured lineages of planctomycete bacteria in these studies suggests that the phylogenetic, metabolic, and physiological diversity of these organisms has not yet been adequately described. The Planctomycetes share a compartmentalized cell plan with the Verrucomicrobia (Fuerst 2005, Lee et al. 2009); this organization appears to be unique among the bacteria. The consequences of this compartmentalization for the biology of the cell remain undetermined, although 
the recent remarkable finding of endocytosis in the planctomycete Gemmata obscuriglobus (Lonhienne et al. 2010) naturally leads to speculation over whether this may occur in other planctomycetes and in Verrucomicrobia. Planctomycetes exhibit other unusual phenotypic properties, such as a cell wall that lacks peptidoglycan, crateriform structures on the cell surface, and diverse cellular appendages (Ward et al. 2006, and the references within). All axenic cultures of planctomycetes are heterotrophic, but the physiology of the uncultivated majority is unclear. There is, however, evidence that planctomycetes possess unique and ecologically important physiologies; for example, certain planctomycete bacteria are uniquely capable of anaerobic ammonium oxidation (anammox reaction) (Strous et al. 1999).

Several lines of evidence suggest that planctomycete bacteria form ecologically relevant associations with algae. The presence of ambient light and development of phototroph populations favor successful laboratory enrichment (Starr \& Schmidt 1989), and freshwater Pirellula spp. are observed as epiphytes of sheathed cyanobacteria (Staley et al. 1992). Kristiansen (1971) suggested that increased iron, manganese, and hydrogen sulfide concentrations resulting from decomposition of phytoplankton support proliferation of Planctomyces bekefii blooms. Blooms of organisms morphologically resembling $P$. bekefii (Schmidt \& Starr 1980) and $P$. guttaeformis have been observed in a Louisiana lake during and subsequent to a bloom of Pithophora spp. (N. L. Ward unpubl. obs.). Budding bacteria resembling planctomycetes were among the dominant organisms enumerated from several lakes in Australia, but were most abundant in eutrophic lakes (Staley et al. 1980). Recently, planctomycete bacteria related to the genus Pirellula were reported to be among the dominant bacteria in an Oregon coast seawater diatom bloom (Morris et al. 2006).

Despite the apparent ubiquity of planctomycetes in aquatic habitats, we lack a detailed understanding of their population diversity and structure. This can be partly attributed to low recovery rates for planctomycete 16S rRNA genes when universal polymerase chain reaction (PCR) primers are employed for sequencing studies. In an attempt to expand recoverable planctomycete diversity, we investigated the use of primers targeting the phylum Planctomycetes. To our knowledge the present study represents the first application of targeted high-throughput pyrosequencing to describe the diversity of planctomycete bacterial populations. Given the reported associations of planctomycetes with eutrophic conditions and algal blooms (described above), we chose to pursue our study in a small eutrophic lake in which an algal bloom was occurring.

\section{MATERIALS AND METHODS}

Sample collection and field measurements. LaBonte Lake, located at $41^{\circ} 19^{\prime} 17.53^{\prime \prime} \mathrm{N}, 105^{\circ} 35^{\prime} 12.96^{\prime \prime} \mathrm{W}$, elevation $2177 \mathrm{~m}$ in Laramie, Wyoming, USA, is a highly impacted urban lake. Samples were collected from LaBonte Lake on June 16, 2008, after the development of macroscopically visible algal mats. The lake has been observed to undergo annual algal blooms that are associated with noxious odors. The algal bloom in 2008 appeared as a mat covering the shallow surface water. Microscopic investigation of the algal mat revealed a community predominantly composed of the filamentous eukaryotic genera Spirogyra and Zygnema, with a minor contribution of Rhizoclonium. A small population of cyanobacterial cells resembling Nostoc was also observed. Algal identification was performed as described by Prescott (1978).

Samples were collected from the lake littoral zone ( $0.5 \mathrm{~m}$ depth) from the south shore of the lake, near the water inlet. This area was free from tree cover, allowing for maximal sun exposure. Water temperature and $\mathrm{pH}$ were measured with an Ecosense water quality monitor (YSI Inc.) and dissolved oxygen was determined with a YSI 55 dissolved oxygen meter (YSI Inc.). Mixed sediment samples were collected with a sterile $50 \mathrm{ml}$ Falcon tube from the upper $10 \mathrm{~cm}$ of sediment and stored in a sterile Whirlpak bag. A $500 \mathrm{ml}$ sample of near-shore lake water (up to $0.5 \mathrm{~m}$ in depth) was collected in a sterile graduated cylinder. This method of water sample collection resulted in a mixing of the water column. Finally, algal mat samples were collected with sterile forceps and placed in $50 \mathrm{ml}$ Falcon tubes. All samples were immediately transported (5 min driving time) to the laboratory for immediate processing.

Laboratory sample processing and chemical analysis. Water samples were filtered through a $2.0 \mu \mathrm{m}$ and then a $0.2 \mu \mathrm{m}$ pore filter, and the $0.2 \mu \mathrm{m}$ retentate was subsequently used for DNA extraction (described in the following subsection). Chemical characterization was performed on the $0.2 \mu \mathrm{m}$ filtrate, which was stored at $-20^{\circ} \mathrm{C}$ until assayed. Total nitrogen, phosphorus (Method 4500-P, B, E) and organic carbon (Method $5310 \mathrm{C}$ ) were analyzed according to standard methods of the American Public Health Association (1995). Total nitrogen was calculated as the sum of total Kjeldahl nitrogen (Method 4500-Norg B) and nitrate (Methods EPA 300.0 \& 300.1). The Wyoming Department of Agriculture Analytical Services performed all chemical analyses.

Community DNA extraction. Total DNA was extracted from a single sample taken from each lake habitat (sediment, water column, and algal mat) using the MoBio PowerSoil kit (MoBio Laboratories), according to the manufacturer's instructions. DNA was ex- 
tracted from $0.5 \mathrm{~g}$ of sediment, $0.2 \mathrm{~g}$ of algal mat material, or the retentate from one-half of the $0.2 \mu \mathrm{m}$ filter. Processing of samples was standardized as much as possible to limit the effects of DNA extraction bias.

Construction of planctomycete 16S rRNA gene clone libraries. Planctomycete 16S rRNA genes were amplified with primers PLA40F (Derakshani et al. 2001) and 1492R (Lane 1991), the same primers used to describe planctomycete-related sequences from soil (Derakshani et al. 2001). The $\sim 1400 \mathrm{bp}$ amplification products were gel purified from $0.8 \%$ agarose gels using the QIAquick kit (Qiagen) with manufacturersupplied protocols. Eluted DNA was cloned into the TOPO-TA vector system (Invitrogen) and transformed into supplied competent DH5 $\alpha$ Escherichia coli cells. Approximately 50 white colonies from each library were selected for plasmid isolation using the Qiagen plasmid mini kit with standard protocols. Sequencing of plasmid DNA was performed with primer PLA40 at the Washington University Genome Sequencing Center.

Editing and analysis of clone library sequences. Sequences from the clone libraries were selected based on 2 criteria: sequence length of at least $500 \mathrm{bp}$ (to ensure overlap with the pyrosequencing results) and quality scores of the sequence. Sequences were edited, and low-scoring bases were trimmed in the Sequencher software package (Gene Codes Corporation). High-quality sequences were aligned using the fast, secondary-structure aware infernal aligner available through the RDP database (Cole et al. 2009) and screened for possible chimeras using the Mallard software package (Ashelford et al. 2006). Potential chimeras were discarded, and the resulting sequences were realigned as above. The sequence alignment was $489 \mathrm{bp}$, and terminal gaps were not considered in the analysis. Sequences were classified using the Greengenes Classify software (DeSantis et al. 2006) and all 3 available models (RDP, NCBI, and Hugenholtz).

Pyrosequencing of planctomycete 16S rRNA genes. Planctomycete tag-encoded FLX-Titanium amplicon pyrosequencing (TETAP) was performed as described previously (Dowd et al. 2008) using the PLA40F and 1492R primers and PCR conditions described above (with the addition of required adaptor sequences). This planctomycete TETAP approach utilized Roche FLX Titanium reagents and procedures and a 1-step PCR. A mixture of Hot Start and HotStar high-fidelity Taq polymerases (Qiagen) was used. Sequencing was performed at the Research and Testing Laboratory (RTL, Lubbock, TX, USA) based upon RTL protocols (www. researchandtesting.com).

16S rRNA gene pyrosequencing analyses. Initial quality filtering, noise removal, and chimera removal were performed on the sequence reads using the Pyro-
Tagger software (Kunin \& Hugenholtz 2010). Further trimming and selection of high-quality sequences were performed using the Mothur software package (Schloss et al. 2009) to select sequences $\geq 200$ bp in length, with no ambiguous bases and no homopolymer stretches of $>7 \mathrm{bp}$. Sequences were aligned over the $200 \mathrm{bp}$, and terminal end gaps were removed from the analysis. Sequence libraries were classified as described for the clone library sequences. After classification, planctomycete-related sequences were collected and retained for further analysis. Planctomycete-related sequences were binned into operational taxonomic units (OTUs) at $97 \%$ sequence identity using the average neighbor algorithm, and these groupings were used for all inter-library comparisons and statistical tests. The $97 \%$ sequence identity threshold was selected because it has long been proposed to represent species-level differences, originally through correlation with genomic DNA:DNA hybridization values (Stackebrandt \& Goebel 1994). It has also been suggested that a $97 \%$ sequence identity threshold minimizes potential inflations of sequence diversity associated with massively parallel tag sequencing (Kunin et al. 2010). Venn diagrams, $H^{\prime}$ (non-parametric), Chaol (Chao et al. 2005), and coverage (Good's coverage) were all calculated within the Mothur software environment, using OTUs defined at $97 \%$ sequence identity (Schloss et al. 2009). These indices were chosen as they are commonly employed in microbial diversity studies and offer a basis for comparison to other ecosystems. LIBSHUFF statistical comparisons were also performed within Mothur using 10000 reiterations.

Phylogenetic analysis of 16S rRNA sequences. For phylogenetic analysis, sequences from the pyrosequencing libraries were aligned against sequences from the clone libraries and clustered into OTUs $(97 \%$ sequence identity) as described above. Representative sequences were extracted from each OTU that was populated by at least 10 sequences, in order to limit the analysis to sequences that were well represented in the library. The representative sequences were then aligned to sequences from type strains of the 11 validly described species within the phylum Planctomycetes, as well as to type strains of representative organisms outside the Planctomycetes but within the Planctomycetes/Verrucomicrobia/Chlamydiae (PVC) superphylum. The 16S rRNA gene sequence of Escherichia coli (ATCC $11775^{\mathrm{T}}$ ) was used as an outgroup to root the tree. Alignments were performed with the RDP as described above. The alignment was used to generate a neighbor-joining tree (BioNJ, observed substitution model) within the SeaView software package (Gouy et al. 2009). The alignment spanned $176 \mathrm{bp}$, corresponding to positions 31 to 212 of the E. coli $16 \mathrm{~S}$ rRNA gene sequence. 
Sequence accession numbers. Planctomycete tag sequences were submitted to the GenBank Short Read Archive and appear under Accession Number SRA012453.6. Clone library sequences were deposited in GenBank and are archived under the Accession Numbers GQ994712 to GQ99493.

\section{RESULTS}

\section{Physical/chemical characterization of the littoral zone of LaBonte Lake}

The physical and chemical characteristics of the littoral zone of LaBonte Lake on June 16, 2008, were as follows: temperature, $25.0^{\circ} \mathrm{C} ; \mathrm{pH}, 9.4$; total nitrogen, $2.1 \mathrm{mg} \mathrm{l}^{-1}$; total phosphorus, $0.13 \mathrm{mg} \mathrm{l}^{-1}$; and total organic carbon, $17.0 \mathrm{mg} \mathrm{l}^{-1}$.

\section{Planctomycete diversity in LaBonte Lake}

We first conducted small-scale cloning-based sequencing to evaluate the effectiveness and specificity of planctomycete-targeted primers. Within the clone libraries produced from the sediment, water column, and algal mat, a total of 133 sequences were recovered, of which 113 (85\%) were identified as being related to the phylum Planctomycetes (Table 1). Nonplanctomycete sequences were related to the bacterial phyla Verrucomicrobia, Lentisphaerae, Chlamydiae, and Proteobacteria (data not shown). The Verrucomi- crobia, Lentisphaerae, and Chlamydiae have been placed in the same superphylum as the phylum Planctomycetes; this group is referred to as the PVC superphylum (Wagner \& Horn 2006). The majority (81) of the 113 planctomycete-related sequences could not be assigned with confidence to any of the known planctomycete genera, but the remaining 32 sequences were affiliated with 5 out of the 9 recognized planctomycete genera (Table 1). The results of this clone library sequencing, therefore, suggested that planctomycetetargeted sequencing, although not perfectly specific, was capable of recovering considerable diversity at the genus level. The estimated coverage of the planctomycete sequence diversity ranged from 7 to $12 \%$ (Table 1), indicating that only a small fraction of the planctomycete diversity in the different environments was described within the small number of clones sequenced. The low coverage obtained with cloningbased sequencing prompted us to next combine the planctomycete-targeted primers with high-throughput pyrosequencing as an alternative sequencing platform.

In total, 19119 16S rRNA gene sequences were obtained from pyrosequencing, of which 6287 were classified as belonging to the phylum Planctomycetes (Table 2). Non-planctomycete sequences were detected at various frequencies in all of the samples. All non-planctomycete sequences were related to the bacterial phyla Actinobacteria, Alphaproteobacteria, BRC1, or to members of the PVC superphylum (data not shown). The estimated coverage of the planctomycete 16S rRNA gene diversity in this pyrosequenc-

Table 1. Description of 16S rRNA gene clone libraries. Good's coverage was calculated using sequences identified as belonging to the planctomycetes, with an operational taxonomic unit definition of $97 \%$ sequence identity

\begin{tabular}{|lcccccccrr|}
\hline Sequences & $\begin{array}{c}\text { Planctomycete- } \\
\text { related } \\
\text { sequences }\end{array}$ & Pirellula & $\begin{array}{c}\text { Blasto- } \\
\text { pirellula }\end{array}$ & $\begin{array}{c}\text { Rhodo- } \\
\text { pirellula }\end{array}$ & $\begin{array}{c}\text { Iso- } \\
\text { Sphaera }\end{array}$ & $\begin{array}{c}\text { Plancto- } \\
\text { myces }\end{array}$ & $\begin{array}{c}\text { Unclassified } \\
\text { Coverage } \\
\%)\end{array}$ \\
\hline Sediment & 46 & 39 & 4 & 1 & 4 & - & 3 & 27 & 9 \\
Water & 44 & 36 & - & - & - & 1 & 1 & 34 & 72 \\
Algal mat & 43 & 38 & 7 & - & 9 & - & 2 & 20 \\
Compiled & 133 & 113 & 11 & 1 & 13 & 1 & 6 & 81 \\
\hline
\end{tabular}

Table 2. Description of 16S rRNA gene pyrosequencing libraries. OTUs: operational taxonomic units; $H^{\prime}$ : Shannon diversity index

\begin{tabular}{|c|c|c|c|c|c|c|c|}
\hline & $\begin{array}{c}\text { Total } \\
\text { sequences }\end{array}$ & $\begin{array}{l}\text { Planctomycete } \\
\text { sequences }\end{array}$ & $\begin{array}{c}\text { Proportion } \\
\text { planctomycetes (\%) }\end{array}$ & OTUs $^{a}$ & $\begin{array}{c}\text { Coverage }^{a} \\
(\%)\end{array}$ & $H^{\prime \mathrm{a}}$ & Chaol $^{\mathrm{a}}$ \\
\hline Sediment & 6552 & 2181 & 33.3 & 1006 & 68.3 & 6.7 & 2610 \\
\hline Water & 7624 & 641 & 8.4 & 337 & 63.1 & 5.8 & 981 \\
\hline Algal mat & 4943 & 3465 & 70.1 & 749 & 86.3 & 5.4 & 1772 \\
\hline Compiled & 19119 & 6287 & 32.8 & 1730 & 81.6 & 6.2 & 4539 \\
\hline
\end{tabular}


ing collection ranged from 63 to $86 \%$, suggesting that the majority of the planctomycete sequences expected to occur in LaBonte Lake were recovered in this survey. Planctomycete-related sequences were assigned to OTUs at $97 \%$ sequence identity, resulting in the identification of a total of 1730 OTUs (Table 2). Based on the ChaoI estimate, over 4500 planctomycete OTUs are expected to be present in LaBonte Lake (Table 2). The highest planctomycete diversity, as described by the Shannon diversity index $\left(H^{\prime}\right)$, was observed in the sediment sample, followed by the water sample, and the lowest diversity was observed in the algal mat (Table 2).

Only 6 OTUs ( $>1 \%$ of planctomycete-related OTUs) showed a common distribution in the lake, in that they contained sequences present in the sediment, water, and algal mat environments, while the majority of OTUs identified in LaBonte Lake appeared to contain sequences unique to a single environment (Fig. 1). Pairwise LIBSHUFF (Schloss et al. 2009) analysis of the sequence libraries indicated a significant difference between the composition of all of the libraries $(p<$ 0.0001 for all combinations with 10000 iterations; data not shown). The clone library sequences were also used as query sequences for a BLASTALL search against the pyrosequencing library. For all but 7 clone library sequences, at least 1 sequence in the pyrosequencing library that was at least $99 \%$ identical to the query sequence was identified. For the remaining sequences, all from the water sample, the identity scores ranged from 88 to $97 \%$ and were within the unclassified sequences (Table 1). This suggests a significant overlap between the sequences reliably classified as planctomycete-related between clone and pyrosequencing libraries, suggesting that the 2 methods primarily differed in their identification of sequences that are potentially non-target organisms.

Within the 6287 planctomycete-related clones obtained from LaBonte Lake, sequences related to all 9 currently described planctomycete genera were identified (Table 3). These included the long-established genera Planctomyces, Pirellula, Gemmata, and Iso-

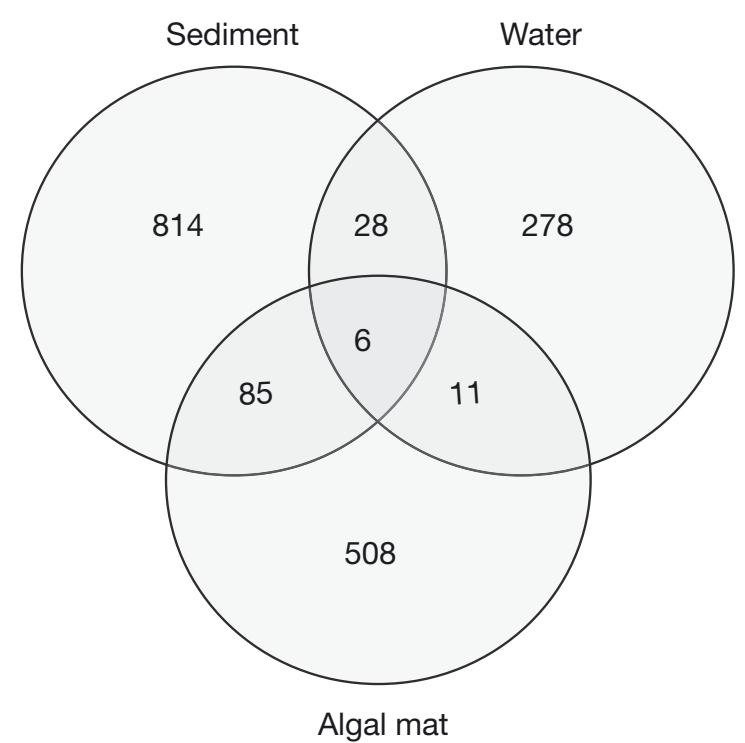

Fig. 1. Operational taxonomic unit (OTU) overlap between different libraries. The number of OTUs unique to each library is indicated within each circle, whereas the numbers of OTUs that were common to multiple libraries are indicated in the overlapping regions. OTU definition $=97 \%$ sequence identity

sphaera, and the more recently reclassified Rhodopirellula and Blastopirellula (formerly contained within Pirellula). Also detected were sequences assigned to the newly described genera Schlesneria (Kulichevskaya et al. 2007), Singulisphaera (Kulichevskaya et al. 2008), and Zavarzinella (Kulichevskaya et al. 2009). Sequences that could not be assigned to any characterized genus were also found in all samples, and in 2 out of 3 sampled lake habitats represented the majority of the planctomycete sequences. Comparison of the planctomycete populations between the different environments suggested a higher relative abundance of the genus Rhodopirellula in the algal mat $(32.7 \%)$ compared to the sediment $(5.5 \%)$ and water $(0.6 \%)$ samples. In the water, $10 \%$ of the sequences were related to the genus Isosphaera, while these organisms

Table 3. Relative abundance (\% of planctomycete sequences) of planctomycete genera within the pyrosequencing libraries. All classifications to the genus level were based on agreement between all 3 models (RDP, Hugenholtz and NCBI) in the Greengenes classifier software; unclassified sequences were all classified as planctomycete bacteria, but were not resolved to genus. Unclass.: unclassified planctomycete; Other: sequences not classified as planctomycete sequences by at least 1 of the models; ND: not detected

\begin{tabular}{|lccccccccccc|}
\hline & $\begin{array}{c}\text { Blasto- } \\
\text { pirellula }\end{array}$ & Gemmata & $\begin{array}{c}\text { Iso- } \\
\text { sphaera }\end{array}$ & Pirellula & $\begin{array}{c}\text { Plancto- } \\
\text { myces }\end{array}$ & $\begin{array}{c}\text { Rhodo- } \\
\text { pirellula }\end{array}$ & Schlesneria & $\begin{array}{c}\text { Singuli- } \\
\text { sphaera }\end{array}$ & $\begin{array}{c}\text { Zavar- } \\
\text { zinella }\end{array}$ & Unclass. Other \\
\hline Sediment & 0.1 & 0.2 & ND & 8.7 & 2.9 & 5.5 & 0.2 & 0.2 & 0.1 & 51.0 & 30.8 \\
Water & ND & ND & 10.0 & 0.9 & 4.1 & 0.6 & 0.2 & ND & ND & 2.3 & 85.9 \\
Algal mat & ND & 0.2 & ND & 5.5 & 1.4 & 32.7 & 0.4 & ND & 0.1 & 52.0 & 7.8 \\
\hline
\end{tabular}


were not detected in either the sediment or algal mat. Sequences allocated to other genera were so similar or rare that comparison of distributions is not expected to be meaningful.

In order to determine the phylogenetic relationships between our lake sequences and described planctomycete species, we constructed a phylogenetic tree containing reference sequences from the 11 validly described planctomycete species and representative sequences from OTUs containing at least 10 sequences (Fig. 2). The tree obtained was constructed from an alignment of only 176 nucleotides, which probably explains some incongruencies between our tree and the established planctomycete phylogeny based on

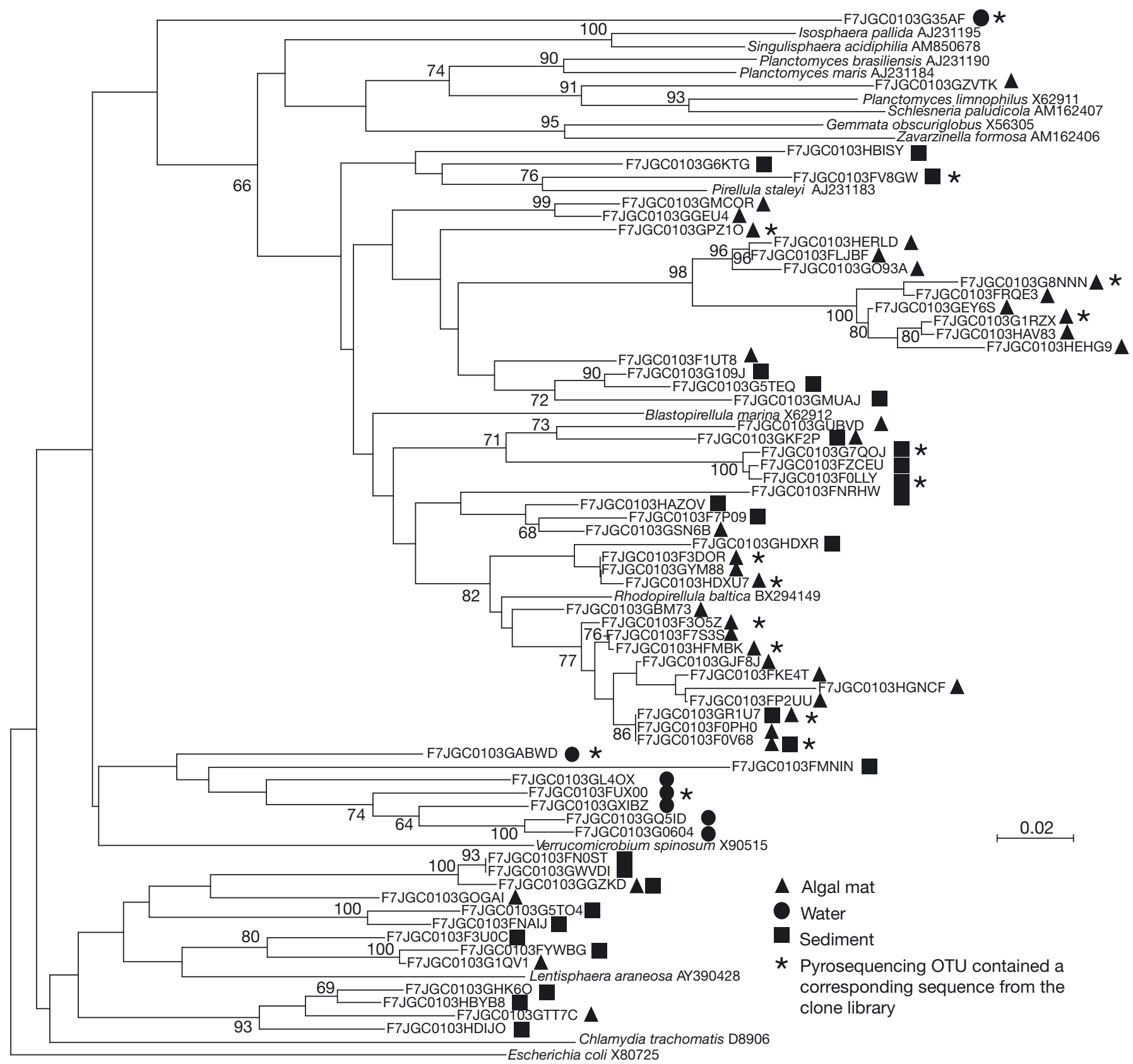

Fig. 2. Neighbor-joining distance tree showing the relationship between representative operational taxonomic unit (OTU) sequences and type strains of related genera. Bootstrap values (1000 resamplings) with $\geq 60 \%$ support are shown at the nodes. Escherichia coli is included as an outgroup to root the tree. Symbols at the leaves indicate the lake environment with which the OTU is associated. In cases where the branch has 2 symbols, the OTU contains sequences from 2 lake environments. Branches labeled with an asterisk indicate OTUs that also include sequences from the clone libraries. Bar $=0.02$ substitutions per site. Gen- 
full-length sequences. For example, we were able to recover the previously reported sister relationships between the genera Isosphaera and Singulisphaera (Kulichevskaya et al. 2008), Gemmata and Zavarzinella (Kulichevskaya et al. 2009), and Planctomyces and Schlesneria (Kulichevskaya et al. 2007). Blastopirellula and Rhodopirellula also clustered with Pirellula, as expected. However, there were some discrepancies in our tree, such as the lack of monophyly for the Planctomyces species, which were interspersed with $S$. paludicola.

Despite these discrepancies, which are probably attributable to the use of short sequences, the phylogenetic relationships of the recovered sequences generally verified the taxonomic classification presented in Tables 1 \& 3, with the majority of sequences clustering with Rhodopirellula, Blastopirellula, and Pirellula species. Few sequences clustered with the type strains from the genera Planctomyces, Gemmata, Singulisphaera, Schlesneria, or Zavarzinella. This was likely due to the low relative abundance of sequences affiliated to these genera in the libraries, leading to their exclusion from the set of OTUs used for phylogenetic reconstruction (i.e. OTUs with $>10$ sequences). In most cases, our lake sequences were not highly related to any of the described, cultured planctomycete species. Several of the clones were more closely related to other sequences from the PVC superphylum than to any planctomycete sequences. On further analysis, these sequences were all within the set of sequences not considered to be planctomycete by at least 1 classifier (Table 3). There was some clustering of planctomycete populations by environment type, although there were also some interspersed sequences from different environments.

\section{DISCUSSION}

To our knowledge, this report presents the largest culture-independent study of planctomycete diversity performed to date. In total 6287 planctomycete sequences were identified via targeted pyrosequencing. These sequences could be assigned to 9 planctomycete genera, indicating the ability of targeted pyrosequencing to recover a diverse planctomycete population. Of these sequences, 3270 (52\%) shared $<97 \%$ sequence identity to any database sequences, suggesting that many originate from previously undescribed planctomycete lineages. It seems likely that subsequent studies employing targeted pyrosequencing could uncover a similar level of planctomycete diversity from other environments. BLASTALL comparison between data obtained from cloning-based and pyrosequencing approaches indicated that the
2 methods detected an overlapping set of planctomycete sequences, with the exception of a small number of clone library sequences that were not detected in the pyrosequencing library. However, the nonoverlapping sequences were all among the sequences that could not reliably be classified to any known planctomycete genus, suggesting that the methods differed mainly in the amplification of organisms distantly related to known planctomycete-related sequences.

Planctomycete-related 16S rRNA genes were identified in all 3 lake environments sampled. However, non-planctomycete sequences were also detected in all of the samples, in both the clone and pyrosequencing libraries. Similar amplification of nontarget organisms with the PLA40 targeted primer has been reported in other studies using clone libraries (Derakshani et al. 2001, Köhler et al. 2008). Currently, we cannot account for the increased detection of nontarget organisms in the pyrosequencing survey relative to the clone library sequencing. Differences in PCR product amplification and purification are inherent in preparing material for the different library constructions and may account for the differences in the ratio of non-target organisms. For example, pyrosequencing requires adding adaptors to the primers during the initial PCR to allow for bead immobilization (http://454.com/products-solutions/how-it-works/ sequencing-chemistry.asp). This modification to the pyrosequencing primer sequence may have slightly altered the thermal properties of the primer as compared to the primers used to generate the clone libraries, accounting for differential amplification. Further studies to characterize these biases will need to be conducted in order to optimize targeted pyrosequencing.

We observed a lack of overlapping OTUs between pyrosequencing libraries obtained from different lake environments (Fig. 1) and clustering of sequences based on the environment of isolation (Fig. 2), suggesting that the populations of planctomycetes differ between the different lake habitats. However, samples from the water column were collected by pre-filtration through $2.0 \mu \mathrm{m}$ pore filters, which would have excluded large particles. Therefore, the planctomycete 16S rRNA gene sequences described in the water likely represent free-living bacterial cells. This physical separation was not applied to the sediment and algal mat samples and so may explain some of the differences seen between the water planctomycete populations compared to the sediment and algal mat populations. The microbial communities associated with particulate matter, or 'lake snow', which were excluded from the present study, have been shown to differ from the free-living microbial community (Riemann \& Winding 2001, Lemarchand et al. 2006). As 
this particulate matter is composed of planktonic or senescent algal cells, bacteria associated with this material may represent an important bacterial-algal association not described in the present study (Tang et al. 2009).

Pyrosequencing suggested an enrichment of sequences related to Rhodopirellula in the algal mat (6-fold greater relative abundance) relative to the other samples. The genome sequence of the marine isolate $R$. baltica encodes a large number of predicted sulfatase genes that may aid in the metabolism of sulfated polymers, such as sulfated fucans, produced by algae (Glöckner et al. 2003). Similar genes may be present in other Rhodopirellula-related organisms. In the water sample, sequences related to Isosphaera were detected, but were not found in the sediment or algal mat. Isosphaera species were first described as thermophiles (Giovannoni et al. 1987), but have also been recovered from non-thermal habitats (Wang et al. 2002). Given that the lake temperature at the time of sampling was $25^{\circ} \mathrm{C}$, Isosphaera sequences recovered in the present study likely represent non-thermophilic organisms. Analysis of a larger number of samples is needed to determine whether these genus-level patterns of distribution are specifically associated with these different habitats.

Our data indicate that pyrosequencing with phylum-targeted primers could be a useful approach for describing the distribution and abundance of organisms that are recalcitrant to laboratory cultivation or are difficult to study due to their relatively low abundance in environmental samples. Refinement of the planctomycete-targeted primers, and determination of the basis for differences between pyrosequencing and clone library sequencing in recovery rates of target organisms, may reduce the recovery of nonplanctomycete sequences and further optimize our ability to describe planctomycete populations in environmental samples. This methodology could be employed for future testing of hypotheses regarding spatial and temporal differences in planctomycete diversity and abundance. Candidate hypotheses arising from this preliminary study include (1) the relative abundance of Rhodopirellula species is higher in algal mats than in other lake habitats; (2) sediments harbor the most diverse planctomycete populations; and (3) most planctomycete OTUs are not shared between habitats.

Acknowledgements. This research was supported by the National Science Foundation (EPS-0447681) and the Wyoming Water Research Program. The Wyoming Department of Agriculture Analytical Services performed water chemical analysis.

\section{LITERATURE CITED}

American Public Health Association (1995) Standard methods for the examination of water and wastewater, 19th edn. APHA, Washington, DC

Andersson AF, Lindberg M, Jakobsson H, Bäckhed F, Nyrén P, Engstrand L (2008) Comparative analysis of human gut microbiota by barcoded pyrosequencing. PLoS ONE 3:e2836

Ashelford KE, Chuzhanova NA, Fry JC, Jones AJ, Weightman AJ (2006) New screening software shows that most recent large 16S rRNA gene clone libraries contain chimeras. Appl Environ Microbiol 72:5734-5741

Chao A, Chazdon RL, Colwell RK, Shen TJ (2005) A new statistical approach for assessing similarity of species composition with incidence and abundance data. Ecol Lett 8:148-159

Chouari R, Paslier DL, Daegelen P, Ginestet P, Weissenbach J, Sghir A (2003) Molecular evidence for novel planctomycete diversity in a municipal wastewater treatment plant. Appl Environ Microbiol 69:7354-7363

Cole JR, Wang Q, Cardenas E, Fish J and others (2009) The ribosomal database project: improved alignments and new tools for rRNA analysis. Nucleic Acids Res 37: D141-D145

Derakshani M, Lukow T, Liesack W (2001) Novel bacterial lineages at the (sub)division level as detected by signature nucleotide-targeted recovery of 16S rRNA genes from bulk soil and rice roots of flooded rice microcosms. Appl Environ Microbiol 67:623-631

DeSantis TZ, Hugenholtz P, Larsen N, Rojas M and others (2006) Greengenes, a chimera-checked 16S rRNA gene database and workbench compatible with ARB. Appl Environ Microbiol 72:5069-5072

Dowd SE, Callaway TR, Wolcott RD, Sun Y, McKeehan T, Hagevoort RG, Edrington TS (2008) Evaluation of the bacterial diversity in the feces of cattle using 16S rDNA bacterial tag-encoded FLX amplicon pyrosequencing (bTEFAP). BMC Microbiol 8:125

Franzmann PD, Skerman V (1984) Gemmata obscuriglobis, a new genus and species of the budding bacteria. Ant Leeuwenhoek 50:261-268

Fuerst JA (2005) Intracellular compartmentation in Planctomycetes. Annu Rev Microbiol 59:299-328

Giovannoni SJ, Schabtach E, Castenholz RW (1987) Isosphaera pallida, gen. and comb. nov., a gliding budding eubacterium from hot springs. Arch Microbiol 147: $276-284$

Glöckner FO, Kube M, Bauer M, Teeling H and others (2003) Complete genome sequence of the marine planctomycete Pirellula sp. Strain 1. Proc Natl Acad Sci USA 100: 8298-8303

Gouy M, Guindon S, Gascuel O (2009) SeaView Version 4: a multiplatform graphical user interface for sequence alignment and phylogenetic tree building. Mol Biol Evol 27: $221-224$

Kahan D (1961) Thermophilic microorganism of uncertain taxonomic status from the hot springs of Tiberias (Israel). Nature 192:1212-1213

Köhler T, Stingl U, Brune A (2008) Novel lineages of Planctomycetes densely colonize the alkaline gut of soil-feeding termites (Cubitermes spp.). Environ Microbiol 10: $1260-1270$

Kristiansen J (1971) On Planctomyces bekefii and its occurrence in Danish lakes and ponds. Bot Tidsskr 66:293-392

Kulichevskaya IS, Ivanova AO, Belova SE, Baulina OI and others (2007) Schlesneria paludicola gen. nov., sp. nov., 
the first acidophilic member of the order Planctomycetales, from Sphagnum-dominated boreal wetlands. Int J Syst Evol Microbiol 57:2680-2687

Kulichevskaya IS, Ivanova AO, Baulina OI, Bodelier PLE, Damsté JSS, Dedysh SN (2008) Singulisphaera acidiphila gen. nov., sp. nov., a non-filamentous, Isosphaera-like planctomycete from acidic northern wetlands. Int J Syst Evol Microbiol 58:1186-1193

Kulichevskaya IS, Baulina OI, Bodelier PLE, Rijpstra WIC, Damsté JSS, Dedysh SN (2009) Zavarzinella formosa gen. nov., sp. nov., a novel stalked, Gemmata-like planctomycete from a Siberian peat bog. Int J Syst Evol Microbiol 59:357-364

Kunin V, Hugenholtz P (2010) PyroTagger: a fast, accurate pipeline for analysis of rRNA amplicon pyrosequence data. The Open Journal 2010, available at www. theopenjournal.org/toj_articles/1

Kunin V, Engelbrektson A, Ochman H, Hugenholtz P (2010) Wrinkles in the rare biosphere: pyrosequencing errors can lead to artificial inflation of diversity estimates. Environ Microbiol 12:118-123

Lane DJ (1991) 16S/23S rRNA sequencing. In: Stackebrandt E, Goodfellow M (eds) Nucleic acids techniques in bacterial systematics. J Wiley \& Sons, Hoboken, p 115-175

Lee KC, Webb RI, Fuerst JA (2009) The cell cycle of the planctomycete Gemmata obscuriglobus with respect to cell compartmentalization. BMC Cell Biol 10:4

Lemarchand C, Jardillier L, Carrias JF, Richardot M, Deboras D, Sime-Ngando T, Amblard C (2006) Community composition and activity of prokaryotes associated to detrital particles in two contrasting lake ecosystems. FEMS Microbiol Ecol 57:442-451

Lonhienne TGA, Sagulenko E, Webb RI, Lee KC and others (2010) Endocytosis-like protein uptake in the bacterium Gemmata obscuriglobus. Proc Natl Acad Sci USA 107: $12883-12888$

Morris RM, Longnecker K, Giovannoni SJ (2006) Pirellula and OM43 are among the dominant lineages identified in an Oregon coast diatom bloom. Environ Microbiol 8: 1361-1370

Prescott GW (1978) How to know the freshwater algae. WCB/McGraw-Hill, Boston, MA

Riemann L, Winding A (2001) Community dynamics of freeliving and particle-associated bacterial assemblages during a freshwater phytoplankton bloom. Microb Ecol 42: 274-285

Schlesner H, Stackebrandt E (1986) Assignment of the genera Planctomyces and Pirella to a new family Planctomycetaceae fam. nov., and description of the order Planctomycetales ord. nov. Syst Appl Microbiol 8:174-176

Schloss PD, Westcott SL, Ryabin T, Hall JR and others (2009) Introducing mother: open-source, platform-independent,

Editorial responsibility: Jed Fuhrman,

Los Angeles, California, USA community-supported software for describing and comparing microbial communities. Appl Environ Microbiol 75:7537-7541

Schmidt JM, Starr MP (1980) Some ultrastructural features of Planctomyces bekefii, morphotype I of the BlastocaulisPlanctomyces group of budding and appendaged bacteria. Curr Microbiol 4:189-194

Stackebrandt E, Goebel BM (1994) Taxonomic note: a place for DNA-DNA reassociation and 16S rRNA sequence analysis in the present species definition in bacteriology. Int J Syst Bacteriol 44:846-849

Staley JT (1973) Budding bacteria of the Pasteuria-Blastobacter group. Can J Microbiol 19:609-614

Staley JT, Marshalland KC, Skerman BD (1980) Budding and prosthecate bacteria from freshwater habitats of various trophic states. Microb Ecol 5:245-251

Staley JT, Fuerst JAF, Giovannoni S, Schlesner H (1992) The order Planctomycetales and the genera Planctomyces, Pirellula, Gemmata, and Isosphaera. In: Balows A, Trueper HG, Dworkin M, Harder W, Schleifer KH (eds) The prokaryotes. Springer, New York, NY, p 3710-3731

Starr MP, Schmidt JM (1989) Genus Planctomyces Gimesi, 1924. In: Staley JT, Bryant MP, Pfennig N, Holt JG (eds) Bergey's manual of systematic bacteriology. Williams and Wilkins, Baltimore, MD, 1946-1958

Steven B, Briggs G, McKay CP, Pollard WH, Greer CW, Whyte LG (2007) Characterization of the microbial diversity in a permafrost sample from the Canadian high Arctic using culture-dependent and culture-independent methods. FEMS Microbiol Ecol 59:513-523

Strous M, Fuerst JA, Kramer EH, Logemann S and others (1999) Missing lithotroph identified as a new planctomycete. Nature 400:446-449

Tang X, Gao G, Qin B, Zhu L, Chao J, Wang J, Yang G (2009) Characterization of bacterial communities associated with organic aggregates in large, shallow, eutrophic freshwater lake (Lake Taihu, China). Microb Ecol 58:307-322

Wagner M, Horn M (2006) The Planctomycetes, Verrucomicrobia, Chlamydiae and sister phyla comprise a superphylum with biotechnological and medical relevance. Curr Opin Biotechnol 17:241-249

Wang J, Jenkins C, Webb R, Fuerst JA (2002) Isolation of Gemmata-like and Isosphaera-like Planctomycete bacteria from soil and freshwater. Appl Environ Microbiol $68: 417-422$

Ward N, Staley J, Fuerst JA, Giovannoni S, Schlesner H, Stackebrandt E (2006) The order Planctomycetales, including the genera Planctomyces, Pirellula, Gemmata, and Isosphaera and the candidatus genera Brocadia, Kuenenia and Scalindua. In: Dworkin M (ed) The prokaryotes, 3rd edn. Springer-Verlag, New York, NY, p 757-793

Submitted: August 10, 2010; Accepted: March 17, 2011

Proofs received from author(s): June 4, 2011 